Artículos Científicos

\title{
Cambio climático: Una percepción de los productores de maíz de temporal en el estado de Tlaxcala, México
}

\section{Climate Change: A Perception of Maize Producers of the State of Tlaxcala, México}

Mudança climática: uma percepção dos produtores sazonais de milho no estado de Tlaxcala, México

Hermila Orozco Bolaños Universidad Autónoma de Tlaxcala, Facultad de Agrobiología, Tlaxcala, México emy_r1@yahoo.com https://orcid.org/0000-0001-5519-1893

Maricela Hernández Vázquez Universidad Autónoma de Tlaxcala, Facultad de Agrobiología, Tlaxcala, México mariheva@live.com.mx https://orcid.org/0000-0003-4562-2324

Guillermina García Juárez Universidad Autónoma de Tlaxcala, Facultad de Agrobiología, Tlaxcala, México nefertiti_ggj58@yahoo.com https://orcid.org/0000-0003-4943-454X

Gerardo Suárez González El Colegio de Tlaxcala, Tlaxcala, México gersuaovis@hotmail.com https://orcid.org/0000-0002-8452-1625 


\section{Revista Iberoamericana de las Ciencias Biológicas y Agropecuarias}

\section{Resumen}

La actividad agrícola en el estado de Tlaxcala depende en gran medida del clima. El productor de maíz se encuentra inmerso en esta contingencia: lo percibe de forma positiva o negativa dependiendo de los sucesos que se presenten. El objetivo fue determinar la percepción y caracterizar los saberes que tienen acerca del clima, el impacto del cambio climático en la producción y la capacidad adaptativa del maíz. La investigación fue de tipo descriptivo-analítico. La población de estudio fueron productores de maíz criollo bajo condiciones de temporal. Se tomó una muestra no probabilística de tipo incidental. La técnica fue la encuesta: se aplicaron 1930 cuestionarios en 39 municipios del estado de Tlaxcala. Los principales hallazgos fueron que $78 \%$ de los productores son mayores de 50 años de edad y $60 \%$ cuenta con educación primaria. La sequía y las heladas son los principales eventos del clima que han impactado al cultivo y han ocasionado pérdidas y bajos rendimientos. Las estrategias que han adoptado ante las variaciones del clima son el cambio en las fechas de siembra, selección de semilla nativa resistente a la sequía y uso de abonos orgánicos para minimizar los efectos del clima. La percepción de los entrevistados estuvo significativamente correlacionada $(p \geq 0.05)$. Los conocimientos y saberes del clima y la semilla que posee el agricultor están en peligro, por lo tanto, la seguridad alimentaria se ve amenazada por los efectos del cambio climático en la producción de maíz de temporal.

Palabras clave: cambio climático, maíz, percepción, productor.

\section{Abstract}

Agricultural activities in the state of Tlaxcala rely mostly on climate conditions. Corn producers know this and adopt a positive or negative perspective according to the way they are present each year. The aim of this work was to determine the producers' perspective and the characterization of knowledge that they possess about weather, climate change impact on production and the adaptability of corn. This research was descriptive-analytical type. The target subject were Creole corn producers under rainfed conditions. It was taken a probabilistic, incidental sample. The used technique was the survey, applying 1930 questionnaires in 39 municipalities in Tlaxcala. From this, it was obtained that $78 \%$ of the interviewed producers are over 50 years old, and $60 \%$ reached primary school education. Drought and frost are the main climate events that have an impact on their crops resulting in low production as well as a great economical lost. Corn producers have developed several strategies due to these climate variations, some of them are: changing planting 
dates, using native seeds resistant to drought, and using organic fertilizers to reduce climatic effects. The interviewed producers' perception was considerably correlated $(p \geq .05)$. It can be perceived from this that the knowledge related to weather conditions and the seeds that farmers have, both are endangered; therefore, food security is threatened due to climatic changes that affect water-fed corn production.

Keywords: climatic change, corn, perception, producer.

\section{Resumo}

A atividade agrícola no estado de Tlaxcala depende em grande parte do clima. O produtor de milho está imerso nessa contingência: ele percebe isso de forma positiva ou negativa, dependendo dos eventos que ocorrem. O objetivo foi determinar a percepção e caracterizar o conhecimento que possuem sobre o clima, o impacto das mudanças climáticas na produção e a capacidade adaptativa do milho. A investigação foi descritivo-analítica. A população do estudo foi produtores de milho crioulo em condições de sequeiro. Uma amostra não probabilística do tipo incidental foi tomada. A técnica foi a pesquisa: 1930 questionários foram aplicados em 39 municípios do estado de Tlaxcala. As principais conclusões foram que $78 \%$ dos produtores têm mais de 50 anos de idade e $60 \%$ têm educação primária. A seca e a geada são os principais eventos climáticos que afetaram a safra e causaram perdas e baixos rendimentos. As estratégias adotadas frente às variações climáticas são a mudança nas datas de semeadura, a seleção de sementes nativas resistentes à seca e o uso de fertilizantes orgânicos para minimizar os efeitos do clima. A percepção dos entrevistados foi significativamente correlacionada $(\mathrm{p} \geq 0,05)$. O conhecimento e conhecimento do clima e da semente que o agricultor tem está em perigo, portanto, a segurança alimentar está ameaçada pelos efeitos das mudanças climáticas na produção de milho de sequeiro.

Palavras-chave: mudança climática, milho, percepção, produtor. 


\section{Introducción}

La agricultura es la actividad en la cual el hombre, en un ambiente dado, maneja los recursos naturales, la calidad y la cantidad de energía disponible y los medios de información para producir y reproducir los vegetales que satisfacen sus necesidades. Se inició con base en una gradual acumulación de conocimiento ecológico y biológico sobre los recursos naturales utilizados; se desarrolló mediante sistemas autóctonos de generación y transmisión de dichos conocimientos, de adaptación y adopción de innovaciones tecnológicas en varias regiones del mundo, entre ellas México (Hernández, 1988).

En nuestro país, la actividad agrícola tiene gran importancia en el medio rural como fuente de ingresos y proveedor de alimentos, misma que depende de los recursos naturales disponibles (Organización de las Naciones Unidas para la Alimentación y la Agricultura [FAO]-Secretaría de Agricultura y Desarrollo Rural [SAGARPA], 2012), y de los elementos del clima como la temperatura y lluvia (Altieri y Nicholls 2009; Hernández, Jiménez, García, Jiménez Orozco, Hernández y Morales 2015), especialmente en la agricultura de temporal. En este sentido, es importante señalar que el planeta tierra, de forma natural y a causa de las actividades antropogénicas, ha presentado cambios en el clima a lo largo de los años, lo que ha dado como resultado el hoy llamado calentamiento global. Esta amenaza la producción de alimentos (Rubio, 2011) a través de olas de calor, sequías, inundaciones, heladas, granizadas y ciclones, exponiendo a los diferentes sistemas como el ecológico y el humano (Conde, Ferrer, Gay y Araujo, 2004; Meza, 2014; Grupo Intergubernamental de Expertos Sobre el Cambio Climático [IPCC], 2014). Existen tres impactos principales del cambio climático en la agricultura: a) deterioro en el rendimiento de los cultivos; $b$ ) efectos en la producción, consumo y comercialización, y $c$ ) efectos en el consumo calórico per cápita y nutrición infantil (FAO y SAGARPA, 2012; Nelson et al., 2009, citados en Meza, 2014; Appendini, 2008; Hernández, García, Orozco y Juárez, 2018).

En México, el cultivo más importante es el maíz, el cual se verá seriamente impactado a raíz del ya mencionado cambio climático en la reducción de áreas potenciales para su producción (Hernández et al., 2018). Frente a este panorama, un personaje determinante es el campesino, quien posee conocimientos y saberes de cuándo sembrar y qué semilla utilizar para enfrentar los siniestros que amenazan los rendimientos necesarios para el autoconsumo. Entre otras problemáticas que enfrentan son el aumento de plagas y enfermedades, lo que provoca impactos negativos sobre su ingreso y seguridad alimentaria en las familias campesinas (FAO y SAGARPA, 2012). Las investigaciones en el tema de percepción del cambio climático sobre el maíz son pocas (Vásquez, 
2016; Ahumada, Velázquez, Flores, y Romero, 2014); existen trabajos realizados en la población indígena y comunidades agrícolas (Barsky, Podestá y Ruiz, 2008; Vander, 2011; Pinilla, Sánchez, Rueda y Pinzón, 2012, Espinoza et al., 2012; Solís y Salvatierra 2013; González et al., 2017), y otros más orientados a la población en general (Oltra, Solá, Sala, Prades y Gamero, 2009; Soares y Gutiérrez 2011; Olmos, Gonzales y Contreras, 2013; Ahumada y García, 2018). En consecuencia, esta investigación se enfoca a la percepción del productor de maíz de temporal, quien enfrenta desafíos al momento de ejercer sus labores, y aplica los conocimientos empíricos adquiridos como estrategias para mitigar los efectos del cambio climático y la seguridad alimentaria.

\section{Método}

\section{Área de estudio}

Esta investigación es de tipo descriptivo-analítico. Para seleccionar la zona de estudio se procedió a regionalizar el estado de Tlaxcala con datos de la superficie sembrada destinada al cultivo de maíz en la modalidad de temporal (Sistema de Información Agroalimentaria y Pesquera [SIAP], 2018).

Los municipios que integraron la zona de estudio fueron 39 de 60. Un criterio de inclusión fue elegir los que contaran con más de 500 hectáreas sembradas de maíz (figura 1). La población de estudio la conformaron productores de maíz en condiciones de temporal. La base de datos se obtuvo del Programa de Apoyos Directos al Campo [Procampo] (2016). En total fueron 26371. De ellos se entrevistó a 1939. La técnica fue la encuesta, el muestreo fue de tipo incidental (Garriga et al., 2015). Se diseñó un cuestionario retomando preguntas relacionadas al cultivo de maíz de Orozco, García, Hernández y Juárez (2016). Asimismo, se elaboraron e incorporaron reactivos referentes al cambio climático. El cuestionario se organizó en cuatro secciones: I. Datos generales, II. Cambio climático y maíz, III. Características y variables en el cultivo de maíz y IV. Maíces que se han perdido. Las respuestas fueron de opción múltiple y abiertas. Cabe subrayar que se realizó una prueba piloto para validarlo. Los datos obtenidos mediante la aplicación del cuestionario se sometieron a una estadística descriptiva para analizar las tendencias de los datos. También se realizó una prueba de Pearson con el software SPSS Versión 21 para determinar si existía correlación entre regiones respecto a la percepción de los productores de maíz en relación con el cambio climático. 
Revista Iberoamericana de las Ciencias Biológicas y Agropecuarias

Figura 1. Regionalización de la superficie sembrada de maíz del estado de Tlaxcala

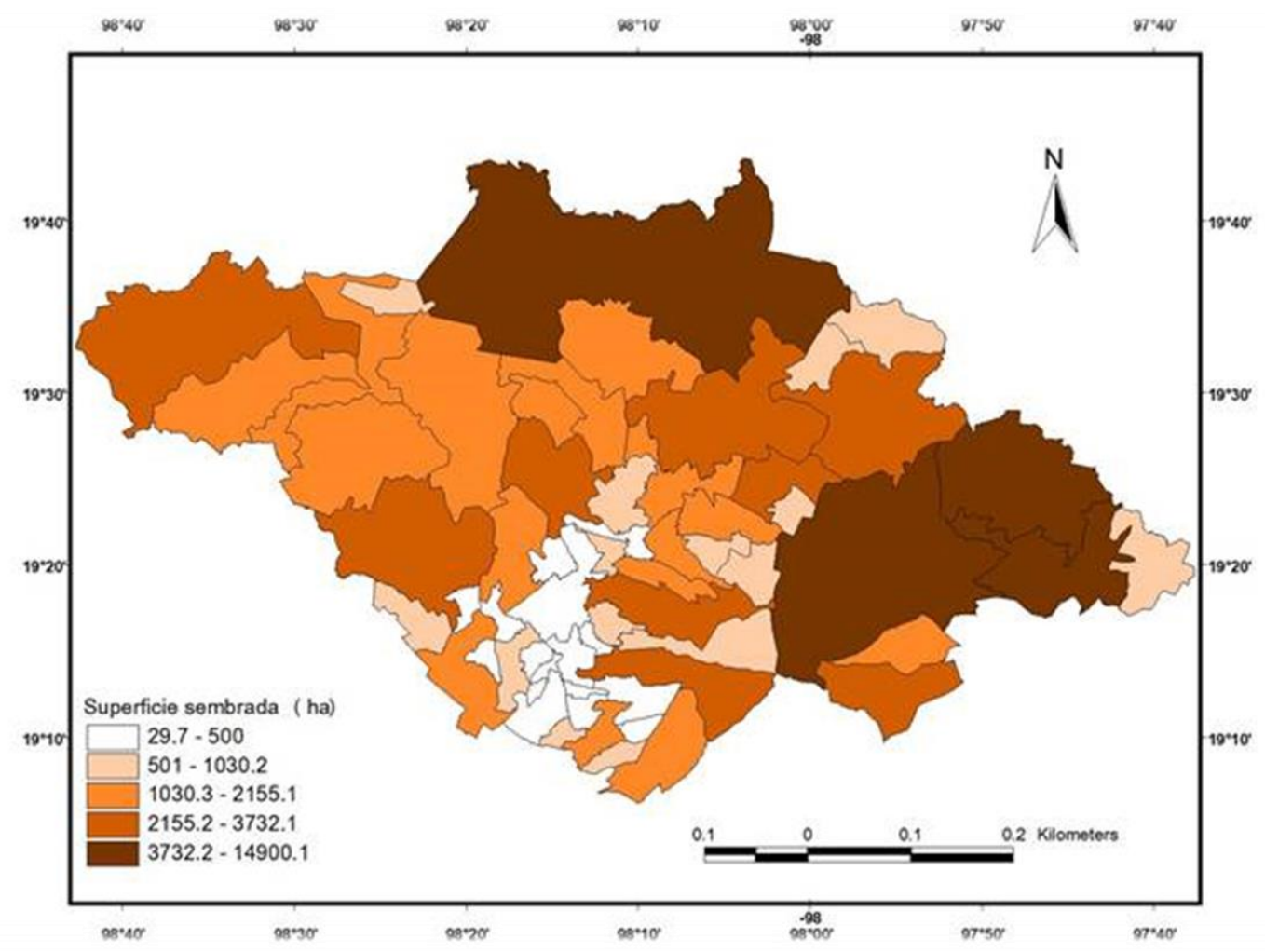

Fuente: Elaboración propia con datos del SIAP (2018)

\section{Resultados}

\section{Datos generales}

Los productores entrevistados que se dedican al cultivo de maíz de temporal tienen una edad promedio de 61 años; 78 \% están en los rangos de 50 a 99 (figura 2). Esto indica que la población es de edad avanzada, los jóvenes no se están integrando al campo. La tendencia de esta situación pone en riesgo no satisfacer la demanda para consumo humano. 
Figura 2. Edad de los productores de maíz del estado de Tlaxcala

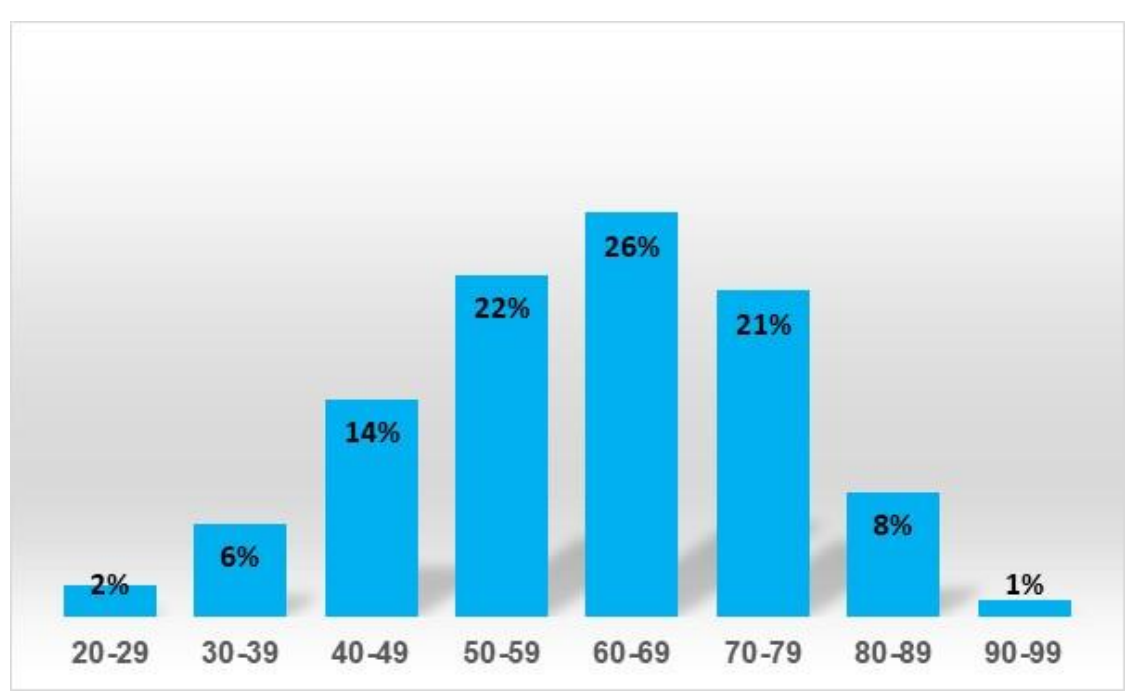

Fuente: Elaboración propia

\section{Producción de maíz}

En el estado, el principal tipo de maíz que siembra el productor es el blanco (55\%), azul (15\%), amarillo (14\%), sangre de cristo (1\%) y otros como el cañuela, rojo grande, pepitilla, moradilla, cacahuacentle y mejorado (15\%). En promedio, la superficie sembrada es de 5.8 ha, con un rendimiento de 2.9 ton/ha. El destino principal de su cosecha es la venta (63\%), para alimento de animales (25\%), para consumo humano (11\%) y para ser guardado parar el siguiente ciclo agrícola (1\%), con esto se mantiene la semilla en los bancos de germoplasma in situ.

\section{Manifestaciones del clima}

En la opinión de los productores, las señales del cambio de clima que se presentan en su comunidad se observan en la figura 3, se destaca el retraso de lluvias (21\%), sequías prolongadas $(18 \%)$ y heladas (16\%). Sin duda estas principales problemáticas observadas son resultado de los efectos del cambio climático, y amenazan la producción en los sistemas agrícolas. 
Figura 3. Señales del cambio de clima en localidades del estado de Tlaxcala

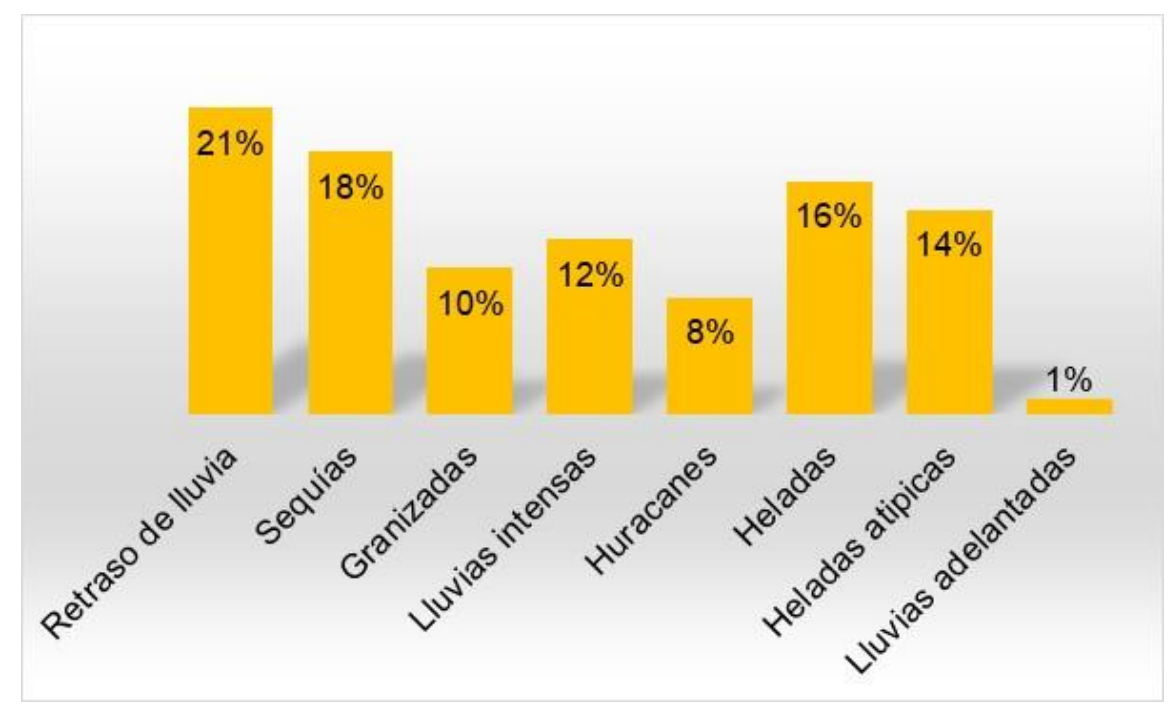

Fuente: Elaboración propia

\section{Presencia de eventos climáticos extremos}

$\mathrm{Al}$ analizar los eventos climáticos y los meses en los que se presentan, se pueden observar cambios de acuerdo con la opinión del productor, por ejemplo, el retraso de lluvias se ha recorrido a mayo y junio. Las sequías se han prolongado a mayo, junio y julio. El mes que presenta el mayor número de granizadas es agosto, aunque también se pueden presentar en junio, julio y septiembre. Las heladas hacen presencia a partir del mes de septiembre (figura 4). Ante esta situación, el productor de maíz se ve frecuentemente amenazado, porque el ciclo agrícola de temporal se ha modificado por esta variabilidad del clima. 
Figura 4. Presencia de los principales eventos climáticos extremos

Retraso de lluvias

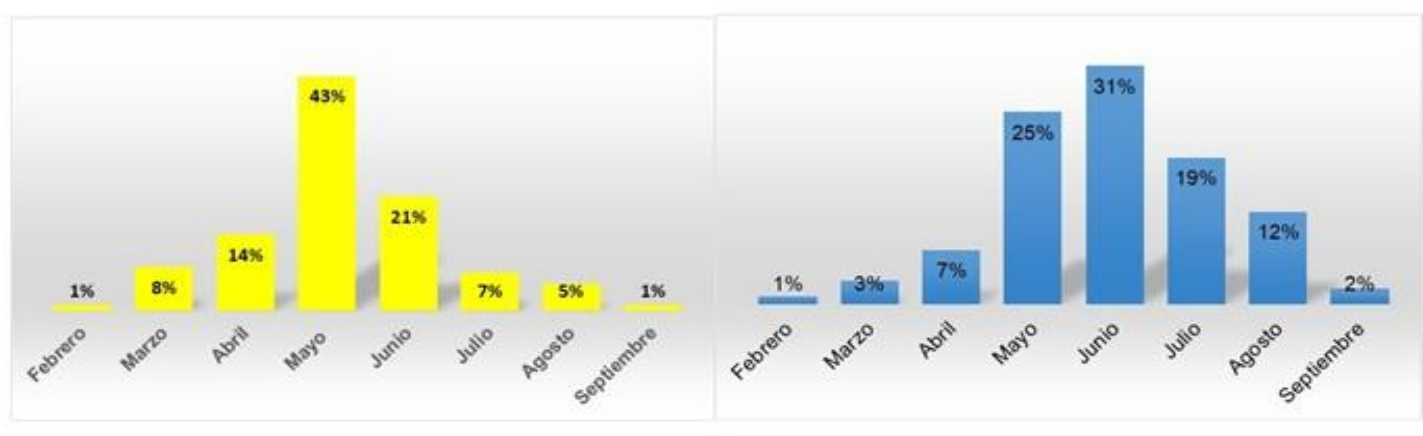

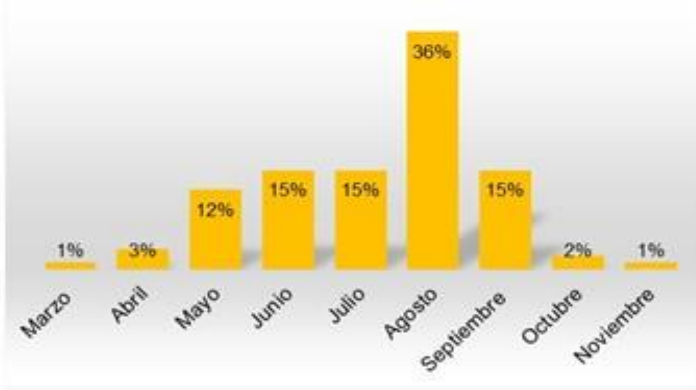

Granizadas

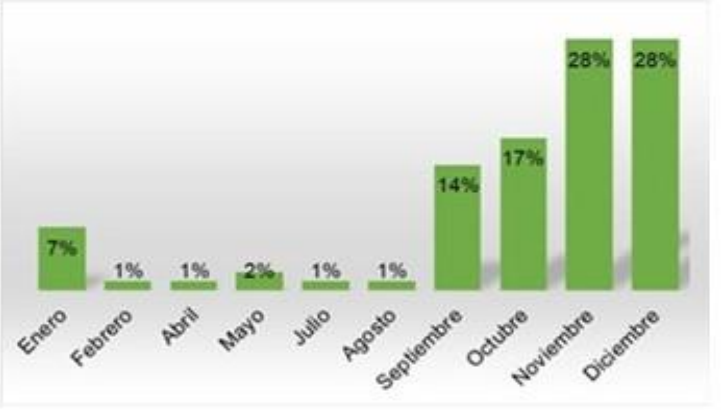

Heladas

Fuente: Elaboración propia

\section{Frecuencia de los eventos climáticos}

En la figura 5 se muestran los eventos climáticos que más afectan el rendimiento de maíz. Se observa que la sequía es el siniestro más frecuente con $40 \%$, seguido de las heladas con $30 \%$. Es claro que el problema es la sequía. Este fenómeno es una tendencia del cambio climático que señala la reducción de lluvias y el aumento de temperatura, y hace vulnerable la producción de maíz. 
Figura 5. Frecuencia de los eventos climáticos que afectan el rendimiento del cultivo de maíz

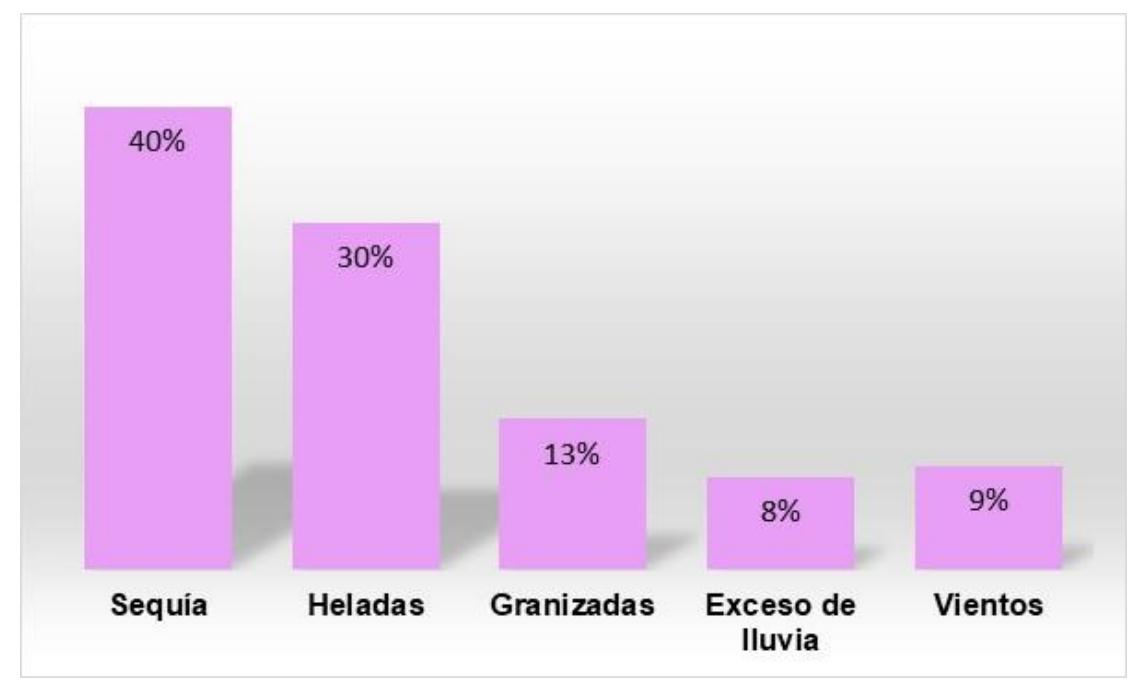

Fuente: Elaboración propia

\section{Rendimiento de maíz}

En la figura 6 se observa el rendimiento de maíz reportado por los productores cuando se presenta sequía o helada. De 39 municipios, 56 \% de ellos son los más vulnerables a las variaciones climáticas, al obtener una producción que va de 93 a 660 kg/ha; Apizaco, Nanacamilpa, Ixtacuixtla, Altzayanca, Xaltocan, Contla, Panotla, Tzompantepec, Tetlanohcan, Muñoz, Teacalco, Teolocholco, Tlaltelulco, Lázaro Cárdenas, Zitlaltepec, Zapata, Yauhquemehcan, Xaloztoc, Tocatlán, Tlaxco, Tetlatlahuca y Tetla son algunos de ellos. Y si bien son estos los que presentaron los rendimientos más bajos, el resto de las localidades también se ve afectado. Lo anterior hace evidente el riesgo en el que se encuentra la seguridad alimentaria para las familias campesinas al ver municipios que están presentando efectos negativos en la producción del maíz. 
Figura 6. Impactos del cambio climático en el rendimiento de maíz

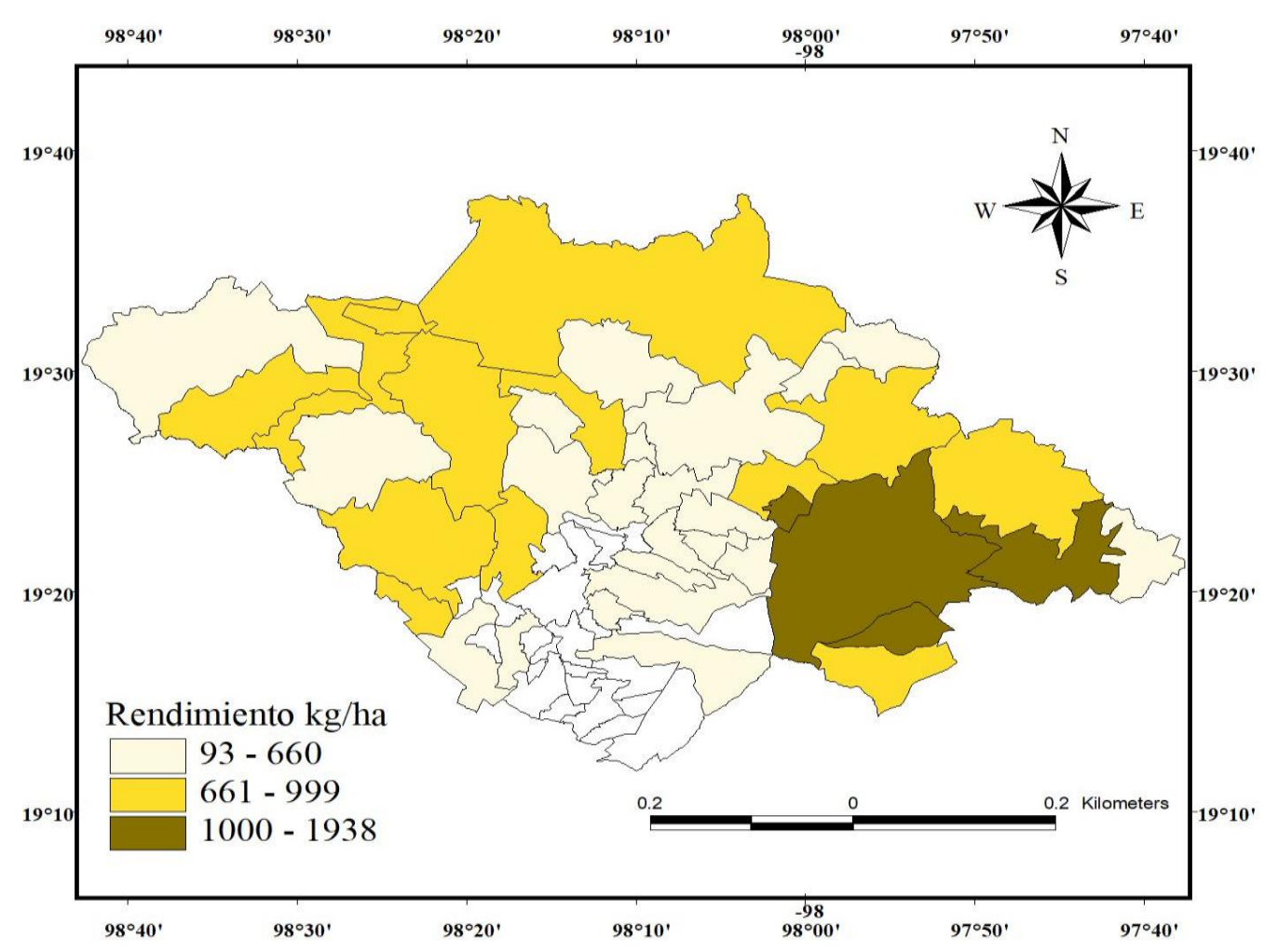

Fuente: Elaboración propia

\section{Estrategias de adaptación}

En la tabla 1 se presentan por municipio las estrategias que han adoptado los agricultores para seguir sembrando maíz ante las variaciones climáticas. De manera general, $28 \%$ ha realizado cambios en las fechas de siembra y selección de semillas nativas resistentes sobre todo a la sequía, seguido de abono orgánico con $26 \%$. Estas son las principales estrategias que se aplican en todos los municipios. Lo cual habla de que el productor está preocupado por las eventualidades climáticas, en especial la sequía, y busca alternativas para seguir produciendo. Es importante señalar que las características para seleccionar la semilla son las siguientes: el tamaño del grano, que no tenga plaga y que esté limpia. Otra estrategia es la aplicación de abono orgánico porque, al aplicarlo, el suelo se enriquece y la planta resiste, hay mayor retención de humedad y se reduce la presencia de plagas. 
Revista Iberoamericana de las Ciencias Biológicas

y Agropecuarias

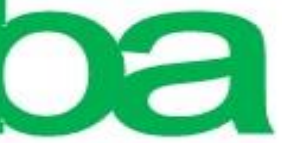

Tabla 1. Estrategias de adaptación ante el cambio climático

\begin{tabular}{|c|c|c|c|c|c|c|}
\hline Municipio & $\begin{array}{c}\text { Cambio } \\
\text { en fechas } \\
\%\end{array}$ & $\begin{array}{c}\text { Semilla } \\
\text { nativa } \\
\%\end{array}$ & $\begin{array}{c}\text { Abono } \\
\text { orgánico } \\
\%\end{array}$ & $\begin{array}{c}\text { Barreras } \\
\text { naturales } \\
\%\end{array}$ & $\begin{array}{c}\text { Variedades } \\
\text { mejoradas } \\
\%\end{array}$ & $\begin{array}{c}\text { Jagüeyes } \\
\%\end{array}$ \\
\hline Altzayanca & 30 & 25 & 28 & 7 & 5 & 4 \\
\hline Apizaco & 27 & 25 & 25 & 14 & 9 & 0 \\
\hline Atlangatepec & 28 & 28 & 25 & 10 & 2 & 6 \\
\hline Benito Juárez & 28 & 26 & 24 & 13 & 6 & 3 \\
\hline Calpulalpan & 27 & 8 & 23 & 17 & 21 & 3 \\
\hline Chiautempan & 29 & 31 & 26 & 12 & 2 & 0 \\
\hline Coaxomulco & 29 & 32 & 27 & 9 & 2 & 1 \\
\hline Contla & 23 & 33 & 33 & 11 & 0 & 0 \\
\hline Cuapiaxtla & 32 & 27 & 26 & 4 & 8 & 2 \\
\hline El Carmen & 29 & 31 & 30 & 0 & 7 & 3 \\
\hline Españita & 28 & 29 & 26 & 9 & 2 & 5 \\
\hline Huamantla & 32 & 27 & 28 & 9 & 1 & 3 \\
\hline Hueyotlipan & 26 & 26 & 24 & 11 & 5 & 7 \\
\hline Ixtacuixtla & 28 & 28 & 27 & 1 & 15 & 1 \\
\hline Ixtenco & 30 & 29 & 27 & 2 & 7 & 4 \\
\hline Lázaro Cárdenas & 28 & 28 & 26 & 10 & 6 & 1 \\
\hline Magdalena & 28 & 32 & 30 & 9 & 1 & 0 \\
\hline Muñoz & 30 & 23 & 25 & 9 & 12 & 0 \\
\hline Nanacamilpa & 27 & 24 & 23 & 11 & 14 & 0 \\
\hline Nativitas & 28 & 30 & 27 & 7 & 7 & 1 \\
\hline Panotla & 31 & 31 & 25 & 5 & 7 & 0 \\
\hline Sanctorum & 27 & 25 & 21 & 15 & 9 & 2 \\
\hline Santa Cruz & 30 & 33 & 29 & 8 & 0 & 0 \\
\hline Teacalco & 25 & 33 & 27 & 13 & 0 & 1 \\
\hline Tecopilco & 28 & 25 & 26 & 13 & 6 & 1 \\
\hline Teolocholco & 28 & 31 & 28 & 11 & 1 & 0 \\
\hline Tepetitla & 29 & 30 & 29 & 5 & 6 & 0 \\
\hline Terrenate & 29 & 26 & 22 & 10 & 9 & 3 \\
\hline Tetla & 27 & 28 & 24 & 9 & 7 & 5 \\
\hline Tetlanhocan & 25 & 31 & 30 & 11 & 2 & 0 \\
\hline Tetlatlahuca & 27 & 30 & 29 & 6 & 8 & 0 \\
\hline Tlaxco & 31 & 27 & 25 & 13 & 1 & 2 \\
\hline Tocatlán & 28 & 30 & 26 & 9 & 5 & 2 \\
\hline Tzompantepec & 30 & 27 & 30 & 9 & 4 & 0 \\
\hline Xaloztoc & 28 & 31 & 27 & 9 & 3 & 1 \\
\hline Xaltocan & 31 & 31 & 27 & 8 & 2 & 0 \\
\hline Yauhquemecan & 30 & 27 & 27 & 10 & 5 & 0 \\
\hline Zapata & 22 & 34 & 26 & 9 & 4 & 4 \\
\hline Zitlaltepec & 28 & 32 & 27 & 11 & 0 & 2 \\
\hline TOTAL & $28 \%$ & $28 \%$ & $26 \%$ & $9 \%$ & $6 \%$ & $2 \%$ \\
\hline
\end{tabular}

Fuente: Elaboración propia 


\section{Percepción del cambio climático}

Con relación a la percepción de los productores, en la tabla 2 se presenta la correlación en las regiones de análisis. Se encontró una correlación significativa en todos los casos $(p \geq 0.05)$ con valores cercanos a uno. Por lo tanto, se evidenció que los efectos del cambio climático han sido percibidos de manera similar por los productores de maíz en los diferentes municipios. Finalmente, señalan que los años en que notaron un cambio radical en el clima de su localidad han sido en los últimos tiempos; 76 \% de los agricultores coincidió en los siguientes años: 1997, 2000, 2002, 2007, 2010, 2011, 2012, 2014, 2015 у 2017.

Tabla 2. Percepción de productores en las regiones de análisis ante el cambio climático

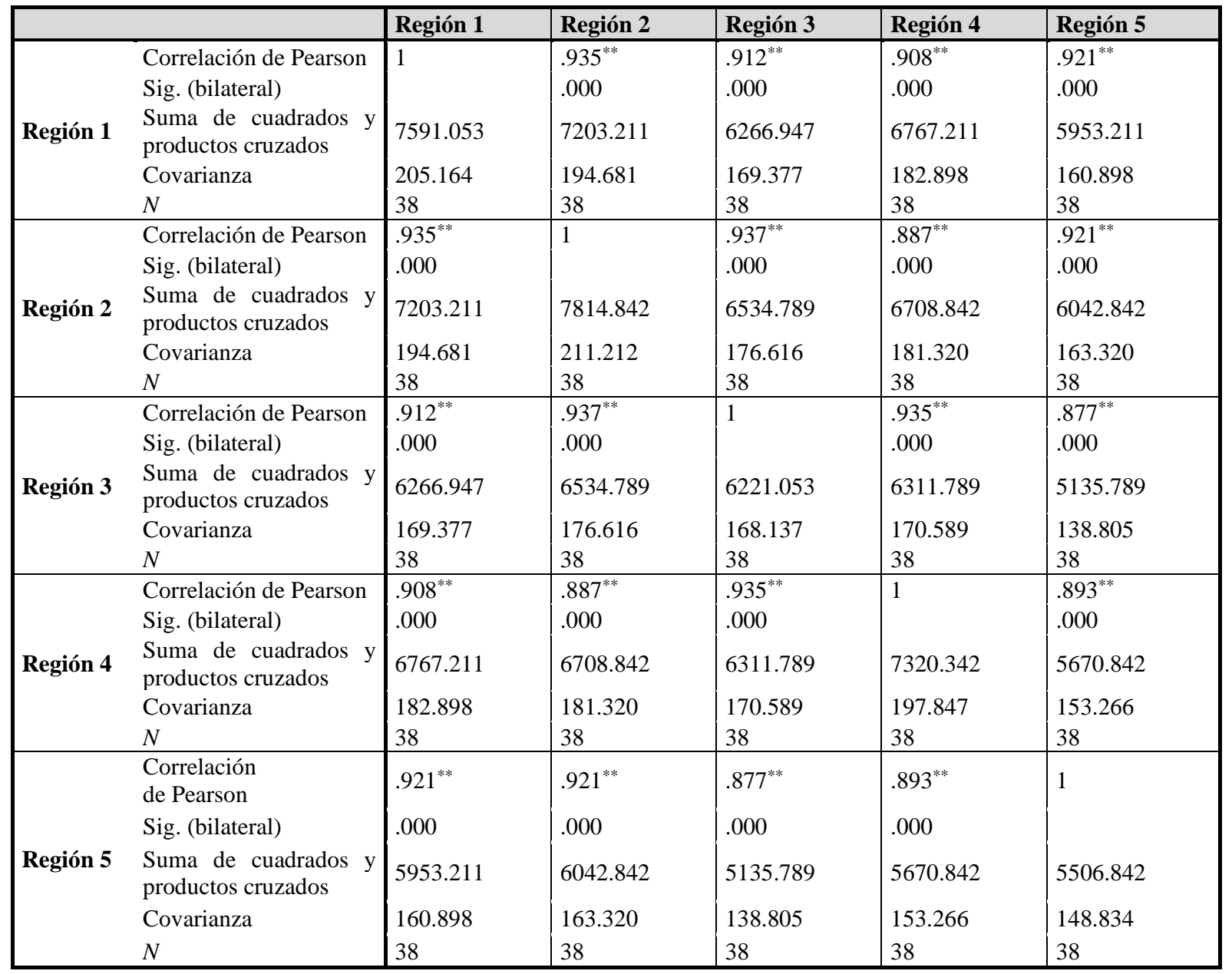

** La correlación es significativa al nivel 0.01 (bilateral)

Fuente: Elaboración propia 


\section{Revista Iberoamericana de las Ciencias Biológicas y Agropecuarias}

\section{Discusión}

El cambio climático es una evidencia del incremento de la temperatura a nivel planetario. Y en los últimos años el sector agrícola ha sido uno de los más afectados por este tipo de alteraciones. Las personas que atienden el campo no son ajenas a estos sucesos. Los productores de maíz perciben dichos cambios en sus localidades, pues impactan el cultivo. Esta aseveración está en sintonía con lo que señala Conde et al. (2004) y Meza (2014): la agricultura es altamente sensible o vulnerable a las variaciones climáticas; la producción de maíz de temporal no es la excepción. Por ejemplo, según lo señalado por Araus, Slafer, Royo y Serret (2008), la falta de agua en el preciso momento de alguna de las etapas de crecimiento de los cultivos limita su productividad. Los productores de maíz son elementos clave para minimizar los impactos del cambio climático en la producción, porque conocen la semilla y su entorno. La experiencia y la observación tienen que ver con el tiempo que le dedican al campo, y esto se relaciona con la edad. Como se ha mencionado líneas arriba, la mayoría son mayores de 60 años. Además, más de la mitad cuenta solo con primaria, información similar a la expuesta por la Comisión Económica Para América Latina y el Caribe [CEPAL] (2014). Este hecho es preocupante porque las nuevas generaciones no se están incorporando al campo (migran para trabajar o estudiar); entonces, no se transmiten los conocimientos empíricos y saberes relacionados con el clima de dicha actividad, lo que pone en riesgo la producción de maíz. Lo anterior sin duda plantea un reto generacional sobre quién va a producir el maíz en el futuro y anticipa, quizá, una dependencia a mayor escala de importaciones transnacionales para alimentarnos.

En México, existen entre 41 y 59 razas de maíz (Reyes, Guerra y Calderón, 2005; Gil y Álvarez 2007; Fitting, 2007; Madrigal, 2008); para el estado de Tlaxcala se reportan 12 razas (María, Hernández, Muñoz de la Vega y Ríos, 2015), lo que representa $24 \%$ a nivel nacional. Lo anterior refiere una riqueza genética de maíz criollo, diferenciado por su color y forma (blanco, amarillo, azul, rojo, negro, rosa, crema, morado, ajo), que se ha conservado por años in situ en los pequeños bancos de almacenamiento (en un cuarto con ventilación dentro de casa, o en zencales — construcciones fuera de casa aproximadamente de $1 \mathrm{~m} \times 1 \mathrm{~m}$, con ventilación—, además de los cuexcomates, entre otros), los cuales preserva el productor al guardar semillas en cada ciclo agrícola. Esta variabilidad de germoplasma es muy importante porque, como lo señalan Altieri y Nicholls (2009) y Cruz (2011), muchas de las comunidades rurales dominadas por la agricultura tradicional se preparan para el cambio climático minimizando las pérdidas en las cosechas mediante un mayor uso de variedades locales de semilla de maíz tolerantes a la sequía. La FAO 
Revista Iberoamericana de las Ciencias Biológicas

y Agropecuarias

(2016) señala que el desempeño agrícola después de eventos climáticos extremos ha demostrado que la resiliencia a los desastres climáticos está vinculada con la diversidad biológica de las regiones. Entonces, la riqueza genética es un elemento importante para mitigar los impactos del cambio climático. El reto es mantener esta diversidad; que no sea sustituida por maíz transgénico (Madrigal, 2008).

Las señales o manifestaciones de algunas variables climáticas que se han modificado en las comunidades aquí estudiadas son el aumento de la temperatura, sequías prolongadas, presencia de heladas, falta de lluvia y granizadas. Estos resultados coinciden con las investigaciones realizadas por Barsky et al. (2008), Oltra et al. (2009), Soares y Gutiérrez (2011), Cruz (2011), Vander (2011), Pinilla et al. (2012), Espinoza et al. (2012), Solís y Salvatierra (2013), Olmos et al. (2013), González et al. (2017), Ahumada y García (2018) y Vásquez (2016), quienes también lo exponen como evidencia de la variabilidad climática que se está presentando, siempre de acuerdo con la percepción de la población. En el caso de Tlaxcala, destacan, en primer lugar, las sequías prolongadas, el retraso de lluvias y heladas, en segundo y tercer lugar respectivamente, variables climáticas que, de acuerdo con el IPCC (2015), son consecuencias de los recientes fenómenos extremos conexos al clima, como olas de calor, sequías, inundaciones, ciclones e incendios forestales, los cuales ponen de relieve una importante vulnerabilidad y exposición de algunos ecosistemas.

Lo anterior se relaciona con la variabilidad climática en el tiempo, por ejemplo, la lluvia ahora se presenta en mayo y junio, y, por lo tanto, la sequía se prolonga a mayo, junio y julio. Hernández et al. (2015) pronostican que si la tendencia de la lluvia es reducirse, entonces aumentarán los periodos de sequías, pero su distribución espacial variará y por ello los impactos serán diferentes entre un lugar y otro. Las heladas se presentan a partir de septiembre y las heladas atípicas en cualquier mes del año. Estos cambios ocasionan que el calendario agrícola se recorra. Castro (2006) compara estos sucesos en el municipio de Calpulalpan, estado de Tlaxcala, en dos periodos 1920-1960 y 1960-2000: la siembra en el primer periodo se realizaba entre abril y mayo; en el segundo, entre junio y julio.

El IPCC (2015) señala que los impactos negativos del cambio climático en el rendimiento de los cultivos han sido más comunes que los impactos positivos. Esto se confirma porque en el caso de los municipios de Tlaxcala, 20 de ellos son las más vulnerables al reportar una producción menor al promedio obtenido (que fue de $689 \mathrm{~kg} / \mathrm{ha}$ ) cuando se presentan siniestros como sequías y heladas. Datos del SIAP (2018) muestran que los años de producción de 1999, 2002, 2005 y 2011 
fueron los más afectados por efectos climáticos, lo que ocasionó que el rendimiento cayera a 1.40, $1.69,1.37,1.64$, respectivamente, muy por debajo de la media nacional ( 2.5 ton/ha). Con esto se reafirma una vez más la vulnerabilidad de los agroecosistemas ante las variaciones climáticas. Meza (2014) asevera que el aumento de temperatura registrado en los últimos 30 años ha generado un descenso del aumento de rendimiento esperado en trigo y maíz. Sin embargo, se puede decir que aun con contingencias climáticas el campesino obtiene producción; hecho que es determinante en la seguridad alimentaria. Por lo anterior, el productor, para afrontar dichas contingencias, implementa alternativas o estrategias para sembrar maíz, tales como el cambio en las fechas de siembra y una selección de semillas nativas resistentes a la sequía, según Altieri y Nicholls (2009) y Barsky et al. (2008). Asimismo, aplican abono orgánico y conservan la semilla in situ, esto es, adaptación al cambio climático (Meza, 2014).

Finalmente, con esta investigación se confirma que, entre las regiones de análisis, la percepción es similar con relación a los efectos del cambio climático, y que en los últimos años los efectos se han acentuado más. Evidentemente estos han obligado a los productores a implementar diversas estrategias para continuar con la producción de maíz y, a la vez, conservar el germoplasma de semillas nativas. Acciones que han coadyuvado a asegurar su disponibilidad. Este último un componente básico de la seguridad alimentaria (Salcedo, 2005).

\section{Conclusiones}

El productor de maíz de temporal es uno de los principales actores en la agricultura y alimentación nacional. Sin embargo, en los municipios de Tlaxcala aquí estudiados, la seguridad alimentaria se ve amenazada porque la producción de maíz de temporal se encuentra básicamente bajo la responsabilidad de productores de edad avanzada. Ellos son los personajes más importantes en la mitigación del impacto del cambio climático por poseer el conocimiento y los saberes del clima.

La biodiversidad de la semilla de maíz es una fortaleza en el campo tlaxcalteca porque está adaptada a las condiciones físicas, geográficas y biológicas, características que le permiten enfrentar las variaciones del clima.

Los eventos climáticos extremos más frecuentes son el retraso de las lluvias, sequías prolongadas y las heladas, los cuales impactan en el rendimiento del maíz de temporal y representan 
un riesgo para el autoconsumo. Ante estos sucesos, la percepción es que sí hay cambios en el clima de las localidades ya especificadas y que los efectos han sido más evidentes en los últimos años.

Las alternativas para mitigar los efectos del cambio climático en la producción de maíz de temporal son principalmente los conocimientos y saberes del productor, que le han permitido implementar diversas estrategias para enfrentar las consecuencias del cambio climático mediante el uso de semilla nativa, cambio de fechas de siembra y la aplicación de abono orgánico.

Este acercamiento con los productores de maíz del estado de Tlaxcala deja aprendizajes muy importantes por lo anterior, este tipo de estudios deben ser considerados en la toma de decisiones para implementar programas y políticas públicas interinstitucionales orientadas hacia la justicia social incluyente; programas que atiendan de forma integral las necesidades en el sector agrícola con un enfoque educativo, económico, cultural, ambiental, laboral, entre otros, tomando como eje indispensable la educación con énfasis en técnicas que puedan ser utilizadas para enfrentar las adversidades del cambio climático, así como información que potencialice las capacidades creativas bajo los principios de la sustentabilidad, encaminadas a preservar las especies de semillas nativas del maíz y los conocimientos y saberes ancestrales que posee el campesino para fortalecer la seguridad y soberanía alimentaria en las localidades y regiones del país.

\section{Agradecimiento}

La autoría agradece al proyecto apoyado por el Fondo Sectorial de la Investigación Ambiental, con clave 263096, SEMARNAT-CONACYT 2015. 


\section{Referencias}

Ahumada, C. R., Velázquez, A. G., Flores, T. E. y Romero, G. J. (2014). Impactos potenciales del cambio climático en la producción de maíz. Investigación y Ciencia, 22(61), 48-53. Recuperado de http://www.redalyc.org/pdf/674/67431579007.pdf.

Ahumada, C. R. y García, L. P. (2018). Conocimiento y percepción acerca del cambio climático en comunidades costeras del municipio de Guasave, Sinaloa, México. Investigación y Ciencia, $\quad 26(75), \quad 38-45 . \quad$ Recuperado de https://investigacion.uaa.mx/RevistaIyC/archivo/rev75.pdf.

Altieri, M. A. y Nicholls, C. I. (2009). Cambio climático y agricultura campesina: impactos y respuestas adaptativas. Agroecología, 5-8. Recuperado de http://socla.co/wpcontent/uploads/2014/leisa-campesino-cambio-climatico.pdf.

Araus, J. L., Slafer, G. A., Royo, C. and Serret, M. (2008). Breeding for yield potential and stress adaptation in cereals. Critical Reviews in Plant Science, 27, 377-412.

Appendini, K., Cortés, L. y Hinojosa, V. (2008). Estrategias de seguridad alimentaria en los hogares campesinos: la importancia de la calidad del maíz y la tortilla. En Appendini, K. y Torres, G. (eds.), Ruralidad sin Agricultura (pp.103-127). Ciudad de México, México: Centro de Estudios Económicos, El Colegio de México.

Barsky, A., Podestá, G. y Ruiz, T. F. (2008). Percepción de la variabilidad climática, uso de información y estrategias de los agentes frente al riesgo. Análisis de esquemas decisionales en agricultores de la región pampeana Argentina. Mundo Agrario, 8(16), 1-42. Recuperado de http://www.redalyc.org/pdf/845/84581603.pdf.

Castro, F. 2006. Colapsos ambientales-transiciones culturales. México: Universidad Nacional Autónoma de México \& Benemérita Universidad Autónoma de Puebla.

Comisión Económica para América Latina y el Caribe [CEPAL]. (2014). Perspectivas de la agricultura y del desarrollo rural en las Américas: Una mirada hacia América Latina y el Caribe 2014. San José, Costa Rica: Comisión Económica para América Latina y el Caribe, Organización de las Naciones Unidas para la Agricultura y la AlimentaciónInstituto Interamericano de Cooperación para la Agricultura.

Conde, C., Ferrer, R. M., Gay, C. y Araujo, R. (2004). Impactos del cambio climático en la agricultura en México. En Martínez, J. y Fernández, A. (coords.), Cambio climático: Una visión desde México (pp. 227-238). México: Semarnat-INE. Recuperado de http://www.data.sedema.cdmx.gob.mx/cambioclimaticocdmx/images/biblioteca_cc/Camb 
io-climatico-una-vision-desde-Mexico-(Julia-Martinez-y-Adrian-Fernandez-Bremauntzcompilado.pdf.

Cruz, L. M (2011). Comparación del ciclo agrícola actual con el de hace unos diez años en san Juan Jalpa municipio San Felipe del progreso estado de México: evidencia de adaptación al cambio climático. Ra Ximhai, 7(1), 95-106. Recuperado de http://www.revistas.unam.mx/index.php/rxm/article/view/26670.

Espinoza, Y., Obispo, N., Gil, J., Rodríguez, M. F., Cortez, A., Rey, J. C., Parra, M. R., Espinoza, F. y Seijas, L. (2012). Percepción del cambio climático en la población rural La Guama, San Sebastián de los Reyes, Aragua Venezuela. Facultad Agronomía, 38(3), 106-114. Recuperado http://www.scielo.org.ar/scielo.php?script=sci_nlinks\&ref=5910830\&pid=S1666$7719201600020000400004 \& \operatorname{lng}=\mathrm{es}$.

Fitting, E. (2007). ¿La economía natural enfrenta a la global? Desafíos a los debates sobre el maíz. Bajo el Volcán, 7(11), 17-44. Recuperado de http://www.redalyc.org/articulo.oa?id=28671103.

Garriga, A. J., Lubin P. P., Merino J. M., Padilla S. M., Recio S. P. y Suárez, J. C. (2015). Introducción al análisis de datos. Madrid, España: Universidad Nacional de Educación a Distancia,

Gil, A. y Álvarez, N. M. (2007). El maíz criollo en la alimentación de las familias campesinas de Santiago Xalitzintla, Puebla. Puebla, México: Colegio de Postgraduados Campus Puebla.

González, L. L., Silva, J. T., Ávila, L. A., Moncayo, E. R., Cruz, C. G. y Ceja, L. F. (2017). El fenómeno de cambio climático en la percepción de la comunidad indígena purépecha del municipio de Chilchota, Michoacán, México. Contaminación. Ambiental, 33(4), 641-653. doi: 10.20937/RICA.2017.33.04.08

Grupo Intergubernamental de Expertos Sobre el Cambio Climático [IPCC]. (2014). El quinto reporte de evaluación del IPCC. ¿Qué implica para Latinoamérica? Resumen ejecutivo. Grupo Intergubernamental de Expertos Sobre el Cambio Climático. Recuperado de https://cdkn.org/wp-content/uploads/2014/12/INFORME-del-IPCC-Que-implica-paraLatinoamerica-CDKN.pdf.

Grupo Intergubernamental de Expertos Sobre el Cambio Climático [IPCC]. (2015). Cambio climático 2014. Informe de síntesis. Ginebra, Suiza: Grupo Intergubernamental de Expertos 
Revista Iberoamericana de las Ciencias Biológicas y Agropecuarias

Sobre el Cambio Climático. Recuperado de https://www.ipcc.ch/site/assets/uploads/2018/02/SYR_AR5_FINAL_full_es.pdf.

Hernández, V. M., Jiménez, G. D., García, J. G., Jiménez, L. J., Orozco, B. H., Hernández, L. M. y Morales, A. T. (2015). Riesgo y vulnerabilidad del maíz de temporal en la región suroeste del estado de Tlaxcala frente al cambio climático. Biológico Agropecuaria Tuxpan, 3(5), 1009-1018. Recuperado de http://revistabioagro.mx/wp-content/uploads/2017/03/Riesgoy-vulnerabilidad-del-ma\%C3\% ADz-de-temporal-en-la-regi\%C3\%B3n-suroeste-delestado-de-Tlaxcala-frente-al-cambio-clim\%C3\%A1tico.pdf.

Hernández, V. M., García, J. G., Orozco, B. H. y Juárez, M.G. (2018). Vulnerabilidad socioambiental del maíz nativo frente al cambio climático en el estado de Tlaxcala, México. Revista iberoamericana de las ciencias biológicas y agropecuarias, 7(14), 53-76.

Hernández, X. E. (1988) La agricultura tradicional en México. Comercio Exterior, 3(8), 673-678. Recuperado de http://revistas.bancomext.gob.mx/rce/magazines/189/2/RCE2.pdf.

Madrigal, A. (2008). Los campesinos se declaran inocentes: Cambios estructurales y contaminación genética del maíz criollo en san Antonio Atotonilco, Municipio de Ixtacuixtla, Tlaxcala. En Ortiz, B. y Duval, G. (coords.), Sistemas complejos medio ambiente y desarrollo (pp. 97-132). México: Lupus Inquisitor.

María, A., Hernández, J. M., Muñoz de la Vega, E. y Ríos, S. A. (2015). Informe final del proyecto el Proyecto FZ016. Conocimiento de la diversidad y distribución actual del maíz nativo y sus parientes silvestres en México segunda etapa 2008-2009. Tlaxcala, México: Comisión Nacional para el Conocimiento y uso de la Biodiversidad. Recuperado de http://www.conabio.gob.mx/institucion/proyectos/resultados/InfFZ016_TLAXCALA.pdf.

Meza, E. L. (2014). La agricultura familiar y el cambio climático. En Organización de las Naciones Unidas para la Alimentación y la Agricultura [FAO], Agricultura familiar en América Latina y el Caribe. Recomendaciones de política (pp. 79-100). Santigo, Chile: Organización de las Naciones Unidas para la Alimentación y la Agricultura. Recuperado de http://www.fao.org/3/i3788S/i3788S.pdf.

Olmos, M. E., Gonzales, M. E. y Contreras, M. R. (2013). Percepción de la población frente al cambio climático en áreas naturales protegidas de Baja California Sur, México. Polis, 12(35), 1-17. Recuperado de https://research.fit.edu/media/sitespecific/researchfitedu/coast-climate-adaptation-library/latin-america-and- 
caribbean/guyana-suriname-fr-guiana/Olmos-Martinez-Et-Alia.-2013.-CC,-PercepcionDe-La-Poblacion.-Baja-California-Sur,-Mexico-[esp].pdf.

Oltra, C., Solá, R., Sala, R., Prades, A. y Gamero, N. (2009). Cambio climático: Percepciones y discursos públicos. Prismasocial, (2), 1-23. Recuperado de http://www.isdfundacion.org/publicaciones/revista/pdf/n2_9.pdf.

Organización de las Naciones Unidas para la Alimentación y la Agricultura [FAO]. (2016). Cambio climático y seguridad alimentaria y nutricional América Latina y el Caribe. (Orientaciones de política). Santiago, Chile: Organización de las Naciones Unidas para la Alimentación y la Agricultura. Recuperado de http://www.fao.org/fileadmin/user_upload/rlc/docs/Cambioclimatico.pdf.

Organización de las Naciones Unidas para la Alimentación y la Agricultura [FAO]-Secretaría de Agricultura y Desarrollo Rural [SAGARPA]. (2012). México: El sector agropecuario ante el desafío del cambio climático (vol. I). México: FAO-SAGARPA Recuperado de http://www.sagarpa.mx/programas2/evaluacionesExternas/Lists/Otros\%20Estudios/Attac hments/37/Cambio\%20Climatico.pdf.

Orozco, B. H., García, J. G., Hernández, V. M. y Juárez, M. G. (2016). Disminución en la frecuencia de uso en maíces de color en dos regiones de Tlaxcala. Biológico Agropecuario Tuxpan, 5(7), 1348-1356. Recuperado de http://revistabioagro.mx/wpcontent/uploads/2016/08/.

Pinilla, M. C., Sánchez, J., Rueda, A. y Pinzón, C. (2012). Variabilidad climática y cambio climático: percepciones y procesos de adaptación espontánea entre campesinos del centro de Santander Colombia. Resultados obtenidos durante el desarrollo de la línea base para el Convenio 46/3379, suscrito entre Isagen E. S. P. y Fundación Natura en el marco del Plan de Manejo Ambiental del Proyecto Hidroeléctrico Sogamoso.

Programa de Apoyos Directos al Campo [Procampo]. (2016). Maíz de temporal. Bases de datos primavera verano 2016, estado de Tlaxcala. Tlaxcala, México: Programa de Apoyos Directos al Campo.

Reyes, G. G., Guerra, N. J. y Calderón, P. G. (2005). Condiciones de cultivo del maíz criollo en comunidades de Puebla, Tlaxcala e Hidalgo: un análisis de las economías de autoconsumo. Aportes, 9(29), 63-82. Recuperado de http://www.eco.buap.mx/aportes/revista/29\%20Ano\%20X\%20Numero\%2029,\%20MayoAgosto\%20de\%202005/06\%20Condiciones\%20de\%20cultivo\%20de\%20maiz\%20criollo 
$\% 20$ en $\% 20$ comunidades $\% 20$ de $\% 20$ Puebla, $\% 20$ Tlaxcala\%20e\%20Hidalgo;\%20un\%20an alisis\%20de\%201as\%20economias\%20de\%20autoconsumo-

Gerando\%20Reyes,\%20Jimena\%20Guerra\%20y\%20Gilberto\%20Calderon.pdf.

Rubio, B. A. (2011). Crisis mundial y soberanía alimentaria en América Latina. Revista de economía mundial, (29), 61-87. Recuperado de https://www.redalyc.org/articulo.oa?id=86622169002.

Salcedo, S. (2005). El marco teórico de la seguridad alimentaria. En Salcedo, S. (ed.), En Políticas de seguridad alimentaria en los países de la Comunidad Andina (pp. 1-8). Santiago, Chile: FAO. Recuperado de http://www.bvsde.paho.org/texcom/nutricion/seguridadCA/03cap1.pdf.

Sistema de Información Agroalimentaria y pesquera [SIAP]. (2018). Anuario estadístico de la producción agrícola. México: Sistema de Información Agroalimentaria y pesquera. Recuperado de www.gob.mx/siap/acciones-yprograma/produccion-agricola-33119.

Soares, D. y Gutiérrez, I. (2011). Vulnerabilidad social, institucional y de percepciones sobre el cambio climático: un acercamiento al municipio de San Felipe, Costa de Yucatán. Ciencia $\begin{array}{lllll}\text { Ergo } & \text { Sum, } & \text { 249-263. } & \text { Recuperado de }\end{array}$ https://dialnet.unirioja.es/servlet/articulo?codigo $=5035053$.

Solís, R. J., De Jesús, R. y Salvatierra, I. B. (2013). Percepción social del cambio climático en áreas destinadas voluntariamente a la conservación en comunidades indígenas de Oaxaca y Chiapas. Temas antropológicos, 35(1), 29-53. Recuperado de http://www.redalyc.org/pdf/4558/455845082002.pdf.

Vander, M. K. (2011). Percepciones de cambio climático y estrategias de adaptación en las comunidades agrícolas de Cotacachi. Ecuador Debate, 145-158. Recuperado de https://repositorio.flacsoandes.edu.ec/bitstream/10469/4263/1/RFLACSO-E82-10VanderMolen.pdf.

Vásquez, M. O. (2016). Percepción del cambio climático por los agricultores de maíz en la zona oriente del estado de Tlaxcala. (tesis). Licenciatura de Biología. Universidad Autónoma de Tlaxcala, México. 
Revista Iberoamericana de las Ciencias Biológicas

y Agropecuarias

\begin{tabular}{|c|c|}
\hline Rol de Contribución & Autor (es) \\
\hline Conceptualización & $\begin{array}{l}\text { Hermila Orozco Bolaños (principal) Maricela Hernández } \\
\text { Vázquez (apoyo) } \\
\text { Guillermina García Juárez (apoyo) }\end{array}$ \\
\hline Metodología & $\begin{array}{l}\text { Hermila Orozco Bolaños (principal) Maricela Hernández } \\
\text { Vázquez (apoyo) } \\
\text { Guillermina García Juárez (apoyo) }\end{array}$ \\
\hline Software & NO APLICA \\
\hline Validación & $\begin{array}{l}\text { Hermila Orozco Bolaños (principal) Maricela Hernández } \\
\text { Vázquez (apoyo) } \\
\text { Guillermina García Juárez (apoyo) }\end{array}$ \\
\hline Análisis Formal & $\begin{array}{l}\text { Hermila Orozco Bolaños (principal) Maricela Hernández } \\
\text { Vázquez (apoyo) } \\
\text { Guillermina García Juárez (apoyo) }\end{array}$ \\
\hline Investigación & $\begin{array}{l}\text { Hermila Orozco Bolaños (principal) Maricela Hernández } \\
\text { Vázquez (apoyo) } \\
\text { Guillermina García Juárez (apoyo) }\end{array}$ \\
\hline Recursos & $\begin{array}{l}\text { Hermila Orozco Bolaños (principal) Maricela Hernández } \\
\text { Vázquez (apoyo) } \\
\text { Guillermina García Juárez (apoyo) Gerardo Suárez González } \\
\text { (apoyo) }\end{array}$ \\
\hline Curación de datos & $\begin{array}{l}\text { Hermila Orozco Bolaños (principal) Maricela Hernández } \\
\text { Vázquez (apoyo) Guillermina García Juárez (apoyo) Gerardo } \\
\text { Suárez González (apoyo) }\end{array}$ \\
\hline $\begin{array}{l}\text { Escritura - Preparación del } \\
\text { borrador original }\end{array}$ & $\begin{array}{l}\text { Hermila Orozco Bolaños (principal) Maricela Hernández } \\
\text { Vázquez (apoyo) } \\
\text { Guillermina García Juárez (apoyo) Gerardo Suárez González } \\
\text { (apoyo) }\end{array}$ \\
\hline $\begin{array}{l}\text { Escritura - Revisión y } \\
\text { edición }\end{array}$ & $\begin{array}{l}\text { Hermila Orozco Bolaños (principal) Maricela Hernández } \\
\text { Vázquez (apoyo) } \\
\text { Guillermina García Juárez (apoyo) Gerardo Suárez González } \\
\text { (apoyo) }\end{array}$ \\
\hline Visualización & $\begin{array}{l}\text { Hermila Orozco Bolaños (principal) Maricela Hernández } \\
\text { Vázquez (apoyo) } \\
\text { Guillermina García Juárez (apoyo) Gerardo Suárez González } \\
\text { (apoyo) }\end{array}$ \\
\hline
\end{tabular}


Revista Iberoamericana de las Ciencias Biológicas y Agropecuarias

\begin{tabular}{|l|l|}
\hline Supervisión & $\begin{array}{l}\text { Hermila Orozco Bolaños (principal) Maricela Hernández } \\
\text { Vázquez (apoyo) } \\
\text { Guillermina García Juárez (apoyo) }\end{array}$ \\
\hline $\begin{array}{l}\text { Administración de } \\
\text { Proyectos }\end{array}$ & $\begin{array}{l}\text { Hermila Orozco Bolaños (principal) Maricela Hernández } \\
\text { Vázquez (apoyo) } \\
\text { Guillermina García Juárez (apoyo) }\end{array}$ \\
\hline Adquisición de fondos & $\begin{array}{l}\text { Hermila Orozco Bolaños (principal) Maricela Hernández } \\
\text { Vázquez (apoyo) } \\
\text { Guillermina García Juárez (apoyo) }\end{array}$ \\
\hline
\end{tabular}

